\title{
A Comparison of DASH Scores Resulting from Different Treatment Options for the Intra-articular Distal Radius Fracture in the Geriatric Population
}

\author{
Himanshu Wagh ${ }^{1}$, Akshay Jakkidi Reddy ${ }^{2}$ \\ ${ }^{1}$ California Northstate University, Rancho Cordova, USA \\ ${ }^{2}$ Department of Ophthalmology, California Northstate University, Rancho Cordova, USA
}

*Corresponding author: Himanshu Wagh, California Northstate University, Rancho Cordova, United States of America, E-mail: Himanshu.wagh5630@cnsu.edu

\section{ABSTRACT}

There exist three main methods of treating distal radius fractures: closed reduction, open reduction internal fixation, and percutaneous fixation. 10 studies in the geriatric population comparing closed reduction and ORIF, and ORIF and methods of percutaneous treatment were found. DASH and PRWE scores from these studies at 1 year followup were compiled and means were compared via independent $T$ tests. DASH scores in the ORIF and percutaneous treatment groups were better than the open reduction, although ORIF and percutaneous treatment groups were not significantly different. PRWE score analysis revealed a significant advantage of ORIF compared to percutaneous treatment, and percutaneous treatment over closed reduction and casting.

\section{INTRODUCTION}

The distal radius fracture has one of the highest incidence rates across all age groups, contributing to $25 \%$ of all fractures in the pediatric population, and $18 \%$ of fractures in the geriatric population [1]. The incidence rate in the United States of America in 2009 was 16.2 per 10,000 people [2]. The average healing time for the fracture is 6 to $8(7.1 \pm 0.9)$ weeks, which is time in which the arm is rendered useless. The importance of the hand is unquestionable, and the loss of hand function can impair patients physically, socially, and psychologically $[3,4]$.

Additionally, it has been shown that orthopedic trauma patients have higher levels of depression post injury, approaching a figure of $45 \%$ in a diverse cohort that one study followed [5]. The personal costs of hand and arm injuries are evident, but the costs to the government are profound as well. Arm and shoulder injuries constitute one of the most expensive worker's compensation claims, averaging $\$ 46,205$ [6]. Depending on the insurance and hospital, the costs of the treatment can also get high.
The high prevalence and profound personal costs of this fracture necessitates a discussion on different treatment methods, and the known benefits or risks of certain procedures. Patients should be able to function well after the fracture is healed, so the treatment methods must be compared to assess their viability and long term benefits.

There are three main treatment options for the distal radius fracture: the closed reduction, the open reduction internal fixation (ORIF), and the percutaneous fixation. Depending on measures such as angulation of the distal fragment, comorbidity of an ulnar fracture, step off distance, and preexisting conditions, the scientific community has some understanding of which procedures should be done. However, consensus has not been completely reached about which procedures must be done when the cases do not fall within currently accepted guidelines.

Research exists comparing the ORIF and the external fixation, and comparing the ORIF and the closed reduction. However, minimal research exists comparing all three treatments in terms of indications, patient outcomes, and price. 
When comparing three distinct procedures and the patient outcomes associated with each, it is essential to narrow down measures of comparison to clear variables that can be measured.

The literature suggests measures of volar tilt, ulnar deviation, and grip strength are indicative of functional outcome [7,8]. However, the metric of grip strength is often given in different ways. Some studies report it as a percentage of the non injured hand, while others give it as a percent change or as an actual value of force. Thus, grip strength is difficult to compare between multiple studies. Using volar tilt as a metric poses similar challenges. Some studies report the actual volar tilt in degrees, while others report percent change. Ulnar deviation, too, is difficult to compare.

So, the primary metrics used to evaluate functional outcomes of the procedures were DASH score and PRWE score. The Disabilities of the Arm, Shoulder and Hand (DASH) outcome measure is a highly standardized self report questionnaire, evaluating difficulties the patient has in various categories such as feeling pain, having difficulties performing certain tasks, and being impacted socially. It is a scale from 0-100, with 0 indicating no disabilities. Because it relies on patient self reporting, it is susceptible to patient based variation. However, large sample sizes are correct for the variation.

PRWE score, or the Patient-Rated wrist evaluation, is also a standardized questionnaire evaluating wrist pain and difficulty with daily tasks. PRWE score is slightly more sensitive to patient differences than the DASH score for particularly distal radius fractures, which is an advantage of evaluating PRWE score [9]. While DASH and PRWE are both reliable and valid, DASH had better test-retest reliability (ICC .91), compared to PRWE (ICC .87) [10]. So, both metrics were used to compare functional outcomes of distal radius fracture treatment methods.

The geriatric population has a high incidence of distal radius fractures due to ground-level falls. As opposed to young adults, distal radius fractures in the geriatric population are much more serious because there are higher rates of osteoporosis [11]. In this population, intra-articular fractures are more common than extra-articular fractures. While intra-articular fractures are generally caused by high impact collisions in younger populations, significantly less force is required for them to occur in the geriatric population, contributing to their high incidence rate [12]. Because of the greater risks present in the geriatric population, this literature review will focus on data for the intra articular distal radius fracture in the geriatric population.

\section{METHODS}

Searches on pubmed were conducted using the keywords "open reduction internal fixation", "external fixation", "closed reduction", "volar locking plate", "K-wire", "percutaneous" "casting", "distal radius", "elderly". Volar locking plate searches yielded information regarding the ORIF, and K-wire and external fixation searches yielded results on the percutaneous fixation. Casting searches showed results concerning closed reductions. To further find studies concerning the topic, the find similar articles feature on PubMed was used. This allowed for the discovery of studies in languages other than english such as French and German. Literature reviews and studies about non-radial fractures were excluded. The data collected specifically concerned the geriatric population. It was made sure that the studies included information on postoperative DASH score or PRWE score, which are two measures that the literature suggest are indicative of functional outcome and are standardized and comparable across studies. These scores were compiled into tables and it was noted whether the studies found a statistically significant difference with $\mathrm{p}<.05$ between the outcomes of the treatment groups. The studies included randomly assigned patients who came to the hospital with distal radius fractures to the treatment groups they were testing: either into closed reduction and ORIF or into ORIF and percutaneous fixation. Discrimination for type of treatment was not made based on radiological outcomes such as ulnar deviation, radial shortening, dorsal tilt, etc. This inclusion criteria kept the DASH and PRWE values impacted only by the type of intervention rather than by the doctor's choice of intervention given certain indications.

This inclusion criteria resulted in the collection of 9 studies comparing DASH scores and 6 studies comparing PRWE scores. The sample sizes of each study, ranging from 38 to 166 , were compiled and are listed in tables 1 and 2 .

All data was retrieved from other studies which had proper patient consent. No personal information was included in this meta analysis, and only values of mean DASH and PRWE scores were retrieved from the studies included.

Once all the DASH and PRWE values were compiled into the tables, averages for each group were found. The calculations used took into account the number of participants per study instead of averaging all studies as if they had the same number of patients. This made sure that studies with high sample sizes were given more weight than studies with only a few participants. 95\% confidence intervals were also found for each group, and independent $\mathrm{T}$ tests comparing groups were performed to find $p$ values to assess significance between the outcomes. $\mathrm{P}$ values resulting from the independent $\mathrm{T}$ tests comparing groups are listed on table 3. 
Table 1: DASH score at 1 year followup

\begin{tabular}{|l|c|c|c|c|c|}
\hline Study & \# Patients & $\mathbf{p}<. \mathbf{0 5}$ & Closed Reduction & ORIF & Percutaneous Fixation \\
\hline Arora 2009 & 130 & no & 11.6 & 11.1 & N/A \\
\hline Arora 2011 & 73 & no & $8.0 \pm 9.3$ & $5.7 \pm 11.1$ & N/A \\
\hline Saving 2019 & 140 & yes & $23.1 \pm 19.8$ & $15.6 \pm 17.0$ & N/A \\
\hline Huard 2010 & 38 & no & N/A & 21 & 17 \\
\hline Jubel 2005 & 55 & no & N/A & 17 & 17 \\
\hline Hollevolet 2011 & 40 & no & N/A & $14 \pm 16$ & $13 \pm 20$ \\
\hline Voigt 2006 & 89 & yes & N/A & 17 & 7 \\
\hline Ma 2016 & 123 & no & N/A & $16.81 \pm 5.98$ & $18.79 \pm 5.54$ \\
\hline Navarro 2016 & 140 & no & N/A & $11 \pm 7$ & $13 \pm 8$ \\
\hline Average: & 828 & & $15.6687 \pm 0.828$ & $13.8155 \pm 0.366$ & $14.1352 \pm 0.517$ \\
\hline
\end{tabular}

Table 2: PRWE score at 1 year followup

\begin{tabular}{|l|c|c|c|c|c|}
\hline Study & \# Patients & $\mathbf{p}<. \mathbf{0 5}$ & Closed Reduction & ORIF & Percutaneous Fixation \\
\hline Arora 2009 & 130 & $\mathrm{n}$ & 16.9 & 9.3 & N/A \\
\hline Arora 2011 & 73 & $\mathrm{n}$ & $14.6 \pm 22.8$ & $12.8 \pm 23.2$ & N/A \\
\hline Saving 2019 & 140 & $\mathrm{y}$ & $22.4 \pm 21.4$ & $12.7 \pm 15.0$ & N/A \\
\hline Lawson 2021 & 166 & $\mathrm{n}$ & 21.5 & 19.8 & N/A \\
\hline Huard 2010 & 38 & $\mathrm{n}$ & $\mathrm{N} / \mathrm{A}$ & 16 & 25 \\
\hline Navarro 2016 & 140 & $\mathrm{n}$ & $\mathrm{N} / \mathrm{A}$ & $13 \pm 9$ & $14 \pm 7$ \\
\hline Average: & 687 & & $19.5929 \pm 0.364$ & $14.0247 \pm 0.384$ & $16.3483 \pm 0.936$ \\
\hline
\end{tabular}

Table 3: statistical significance between group means

\begin{tabular}{|l|c|c|}
\hline Group comparison & DASH p value & PRWE p value \\
\hline 1 vs 2 & $<.001$ & $<.001$ \\
\hline 1 vs 3 & $<.001$ & $<.001$ \\
\hline 2 vs 3 & .312 & $<.001$ \\
\hline
\end{tabular}

\section{RESULTS}

Thus, a comprehensive data pool of 10 studies were obtained for analysis, consisting of data from 828 geriatric patients for DASH score and 687 patients for PRWE score. The average DASH score for closed reduction, ORIF, and percutaneous fixation were $15.6687 \pm 0.828,13.8155 \pm 0.366$, and 14.1352 \pm 0.517 , respectively. The percutaneous fixation group had significantly better outcomes than the closed reduction group $(\mathrm{p}<.001)$, and the ORIF group had significantly better outcomes than closed reduction group as well $(\mathrm{p}<.001)$. However, there was no significant difference between the DASH scores of the ORIF and percutaneous fixation groups $(\mathrm{p}=.312)$. The average PRWE scores for closed reduction, ORIF, and percutaneous fixation were $19.5929 \pm 0.364,14.0247 \pm 0.384$, and 16.3483 \pm 0.936 , respectively. All three groups were significantly different from each other, with ORIF offering the best results, followed by percutaneous fixation, and then by closed reduction. The $\mathrm{p}$ value was $<.001$ for all three comparisons.

\section{DISCUSSION}

The closed reduction does not involve invasive surgery, so it serves as a simple, efficient procedure that can be successful for less serious fractures. It involves injecting a needle into the hematoma, aspirating the blood, injecting a local anesthetic such as lidocaine into the hematoma, applying traction to loosen the muscles, and then manually pushing the distal radius back into alignment with the proximal radius segment [13]. This could be done with the assistance of a fluoroscope to periodically assess bone positioning. Oftentimes, a pop sound can be heard to indicate that proper bone alignment has been achieved. Multiple iterations of applying traction and pushing the bone are often required to reach the desired positioning [14].

It is currently accepted that conservative treatment should be performed when the fracture is nondisplaced, incomplete, extra articular and can be reduced, or if surgery is too dangerous for the patient[15]. If the fracture is unstable, then more complex procedures must be performed. 
One such procedure is percutaneous fixation, which can either be with Kishner wires, or with an external fixator. Kirschner wires are metal rods which are inserted at certain angles to stabilize the bone fragments [16]. A cast can be put on the patient's arm after the K-wires are inserted. Or, an external fixator can be installed. These methods both are minimally invasive, and do not involve open surgery. Instead, the procedures are done by inserting rods through the skin, and into the bone with the case of the external fixation [17]. When the fracture is healed, the rods are removed and the skin and muscles heal.

Some studies claim that this procedure should be performed when there are more than 2 fragments resulting from the fracture that are properly reduced. Another indication is old age, as there is a greater risk of infections resulting from more invasive procedures [18]. It is often used when a simple closed reduction does not completely stabilize the fracture.

However, there is another alternative which is used when closed reduction does not result in a stable fracture. This is the Open Reduction Internal Fixation, or ORIF, which consists of a procedure in which either the dorsal or volar forearm is opened, the muscles are displaced, and either a dorsal or volar locking plate is installed by drilling it into the bone fragments [19].

Dorsal locking plates are less common than volar locking plates, due to their complications with regard to extensor tendon irritation and rupture [20] and higher incidence of volar collapse [21]. These devices are then removed after the fracture is healed, which requires another procedure and further disturbance of the muscles in the area.

When the fracture presents as an open fracture and the skin is already torn, the fracture is most likely serious. Additionally, open fractures offer a location where the volar or dorsal locking plate can easily be inserted with minimal extra damage [15]. They are also done when closed reduction does not restore a congruent articular surface or when there is significant radial shortening of more than $5 \mathrm{~mm}$ [15]. Another study evaluating surgeon views found that there is high consensus among surgeons that an intra-articular step-off of greater than or equal to $2 \mathrm{~mm}$ is an absolute indication for ORIF in patients under 65 years of age [22]. Comorbidity of an ulnar shaft fracture also indicates open surgery, as both fractures are more easily treated that way [15].

No significant difference was found in DASH scores or PRWE scores at the 1 year mark in two studies comparing closed reduction and casting and ORIF, but differences were found at 6 weeks, with the ORIF group having better outcomes with a DASH score of 18.8 vs 34.4 in the closed reduction group [23]. Grip strength was also better in the ORIF group at this time point.
These differences were mitigated past 12 weeks. At the 1 year final assessment, there were no statistically significant differences in any metric [23-24]. However, one other study found ORIF led to significantly better DASH, PRWE, flexion, ulnar deviance, and grip strength when compared to the closed reduction and casting group even at the 1 year followup [25]. These three studies offer contradictory results, even though they are all prospective randomized trials. Arora (2011) had a sample size of 73 patients. Only patients who had an initial inadequate reduction or a loss of reduction at 1 week followup were included in the study, and the mean age was 76.7. Saving (2019) included data of 119 participants at 12 months followup, with an average age of 79 . The most relevant aspect in which these studies differed was the frequencies of the exact type of distal radius fractures. Arora (2011) had high frequencies of type C fractures, with $69.9 \%$ of participants having either type C1, C2, or C3. Saving (2019) had a high frequency of type A2 and A3 fractures at $48.7 \%$ and just $51.3 \%$ being type $\mathrm{C} 1, \mathrm{C} 2$, and C3 fractures. Arora (2009) did not report as much data, and not all the patients came back at the 1 year followup. Instead, some patients came much later, so the data showed less significant differences than would be seen if the patients all came at the 1 year followup. So, it is difficult to assess the efficacy of closed reduction and casting to ORIF for all types of distal radius fractures. Clear results have been shown for the intra-articular fracture though, with ORIF leading to better DASH and PRWE outcomes even at the 2 year followup [26].

The results comparing percutaneous fixation and ORIF in particular are often complex. These two procedures both stabilize unstable fractures post closed reduction, but seem to offer different benefits and disadvantages. One major disadvantage of the ORIF is that it leads to significantly more infections in the geriatric population, necessitating replacement and further infection controlling treatments, which hinder the healing process [18]. In the geriatric population, most studies found no significant difference in DASH and PRWE scores at 1 year post treatment. Even across other metrics such as grip strength, volar tilt, and radial shortening, no statistically significant differences were found. [27]. However, significant differences were noted at time points less than 3 months post treatment. One study found ORIF led to significantly better ulnar variance compared to $\mathrm{K}$-wire treatment at 5 weeks. However, the other measures were not statistically significant at that time onwards [27]. Other studies found ORIF led to better EuroQol-5D scores than K-wires in the first 6 weeks, but did not differ after that point [28].

DASH and PRWE scores were not statistically significant at any point. In the geriatric population, the literature suggests that most metrics are similar after 3 months, and there exist some differences before that point. It is not the same for younger populations. One study found Gartland scores were higher in 
the ORIF group than the K-wire group for patients under 60 years of age [29]. In the geriatric population, the correlation between clinical outcome and anatomical differences is not as pronounced, which may explain the similarities between the functional outcomes found in the studies analyzed in this paper [30]. This may be due to the reduced physical demands in this population compared to younger populations, who tend to be more physically active.

Not all studies supported the statistical insignificance of the outcomes between percutaneous treatment groups and ORIF groups in the elderly. Voigt (2006) found favorable DASH scores in the K-wire group. The study attributed the better DASH scores for the K-wire group to the earlier return to daily activities of living times and the lack of an obligatory second surgery [31]. However, the study only found this was true for type $\mathrm{A} 3$ and $\mathrm{C} 2$ fractures.

Cost is also a consideration when evaluating treatments. One cost analysis study found the average cost of a pack of 10 K-wires was 3 euros in February 2009, or 3.83 US dollars at that time. The cost of the volar locking plate and screws was 787 euros, or 1,004.45 US dollars at that time [32,33]. Adjusted for inflation, the difference between $\$ 1,224.16$ and $\$ 4.67$ is $\$ 1,219.49$ as of January 2021 [34]. Although the prices of these treatments may have changed, just the materials needed for ORIF are still more expensive. This measurement still does not factor in time needed to complete the procedures. The same study found that ORIF took an average of 65 more minutes than the K-wire procedure. K-wire treatment took an average of 56 minutes while ORIF took 121 minutes on average. This leads to greater costs of the surgery as well. Closed reduction takes the least time, at $24 \pm 7.5$ minutes [35]. In a setting such as an emergency room, which could be filled with patients with dire needs, time is also a necessary consideration [36-38].

\section{REFERENCES}

1. Nellans KW, Kowalski E, Chung KC (2012) The epidemiology of distal radius fractures. Hand Clin. 28(2):113-25.

2. Karl JW, Olson PR, Rosenwasser MP (2015) The Epidemiology of Upper Extremity Fractures in the United States, 2009. J Orthop Trauma. 29(8):e242-4.

3. Meyer TM (2003) Psychological aspects of mutilating hand injuries. Hand Clin. 19(1):41-9.

4. Bonanno GA (2004) Loss, trauma, and human resilience: have we underestimated the human capacity to thrive after extremely aversive events? Am Psychol. 59(1):20-8.

5. Crichlow RJ, Andres PL, Morrison SM, Haley SM, Vrahas MS (2006) Depression in orthopaedic trauma patients. Prevalence and severity. J Bone Joint Surg Am. ;88(9):1927-33.
6. Cost of Injury (2020) Sound Ergonomics [cited 2021]. Available from: https://soundergonomics.com/useful-links/ cost-of-injury/

7. Dario P, Matteo G, Carolina C, Marco G, Cristina D, etal. (2014) Is it really necessary to restore radial anatomic parameters after distal radius fractures? Injury. 45 Suppl 6:S21-6.

8. Larouche J, Pike J, Slobogean GP, Guy P, Broekhuyse H, et al. (2016) Determinants of Functional Outcome in Distal Radius Fractures in High-Functioning Patients Older Than 55 Years. J Orthop Trauma. 30(8):445-9.

9. Imaeda $\mathrm{T}$, Uchiyama S, Wada $\mathrm{T}$, Okinaga S, Sawaizumi T, et al. (2010) Clinical Outcomes Committee of the Japanese Orthopaedic Association and the Functional Evaluation Committee of the Japanese Society for Surgery of the Hand. Reliability, validity, and responsiveness of the Japanese version of the Patient-Rated Wrist Evaluation. J Orthop Sci. 15(4):509-17.

10. Kleinlugtenbelt YV, Krol RG, Bhandari M, Goslings JC, Poolman RW, et al. (2018) Are the patient-rated wrist evaluation (PRWE) and the disabilities of the arm, shoulder and hand (DASH) questionnaire used in distal radial fractures truly valid and reliable? Bone Joint Res. 7(1):36-45.

11. Oyen J, Brudvik C, Gjesdal CG, Tell GS, Lie SA, et al. (2011) Osteoporosis as a risk factor for distal radial fractures: a case-control study. J Bone Joint Surg Am. 16;93(4):348-56.

12. Martinez-Mendez D, Lizaur-Utrilla A, de-Juan-Herrero J. (2018) Intra-articular distal radius fractures in elderly patients: a randomized prospective study of casting versus volar plating. J Hand Surg Eur Vol. 43(2):142-147.

13. Vampertzis T, Barmpagianni C, Iosifidou E, Papastergiou S. (2019) Closed Unassisted Reduction in Emergency: A Technique for Unassisted Closed Reduction of Colles Fractures Without Equipment. J Emerg Med. 7:S07364679(19)30931-X.

14. Schermann H, Kadar A, Dolkart O, Atlan F, Rosenblatt Y, et al. (2018) Repeated closed reduction attempts of distal radius fractures in the emergency department. Arch Orthop Trauma Surg. 138(4):591-596.

15. Tang JB (2014) Distal radius fracture: diagnosis, treatment, and controversies. Clin Plast Surg. 41(3):481-99.

16. Gibbs J, Maclean A, Ricketts D (2006) K-wiring distal radial fractures, an alternative technique. Ann R Coll Surg Engl. 88(2):227-8.

17. Capo JT, Swan KG Jr, Tan V (2006) External fixation techniques for distal radius fractures. Clin Orthop Relat Res. 445:30-41.

18. Ma C, Deng Q, Pu H, Cheng X, Kan Y, et al. (2016) External fixation is more suitable for intra-articular fractures of the distal radius in elderly patients. Bone Res. 21;4:16017. 
19. Nydick JA, Streufert BD, Stone JD (2017) Intraarticular Distal Radius Fracture Open Reduction Internal Fixation. J Orthop Trauma. 31 Suppl 3:S45-S46.

20. Kambouroglou GK, Axelrod TS (1998) Complications of the AO/ASIF titanium distal radius plate system (pi plate) in internal fixation of the distal radius: a brief report. J Hand Surg Am. 23(4):737-41.

21. Ruch DS, Papadonikolakis A (2006) Volar versus dorsal plating in the management of intra-articular distal radius fractures. J Hand Surg Am. 31(1):9-16.

22. Mulders MA, Rikli D, Goslings JC, Schep NW (2017) Classification and treatment of distal radius fractures: a survey among orthopaedic trauma surgeons and residents. Eur J Trauma Emerg Surg. 43(2):239-248.

23. Arora R, Lutz M, Deml C, Krappinger D, Haug L, et al. (2011) A prospective randomized trial comparing nonoperative treatment with volar locking plate fixation for displaced and unstable distal radial fractures in patients sixty-five years of age and older. J Bone Joint Surg Am. 93(23):2146-53.

24. Arora R, Gabl M, Gschwentner M, Deml C, Krappinger D, et al. (2009) A comparative study of clinical and radiologic outcomes of unstable colles type distal radius fractures in patients older than 70 years: nonoperative treatment versus volar locking plating. J Orthop Trauma. 23(4):237-42.

25. Saving J, Severin Wahlgren S, Olsson K, Enocson A, Ponzer S, et al. (2019) Nonoperative Treatment Compared with Volar Locking Plate Fixation for Dorsally Displaced Distal Radial Fractures in the Elderly: A Randomized Controlled Trial. J Bone Joint Surg Am. 5;101(11):961-969.

26. Martinez-Mendez D, Lizaur-Utrilla A, de-Juan-Herrero J. (2018) Intra-articular distal radius fractures in elderly patients: a randomized prospective study of casting versus volar plating. J Hand Surg Eur Vol. 43(2):142-147.

27. Hollevoet N, Vanhoutie T, Vanhove W, Verdonk R. (2011) Percutaneous K-wire fixation versus palmar plating with locking screws for Colles' fractures. Acta Orthop Belg. 77(2):180-7.

28. Mellstrand Navarro C, Ahrengart L, Törnqvist H, Ponzer S. (2016) Volar Locking Plate or External Fixation With Optional Addition of K-Wires for Dorsally Displaced Distal Radius Fractures: A Randomized Controlled Study. J Orthop Trauma. 30(4):217-24.

29. Jubel A, Prokop A, Andermahr J, et al. (2005) Functional Outcome Following Fixed-Angle Volar Plating or Intrafocal
K-Wire Fixation for Extraarticular Fractures of the Distal Part of the Radius. Eur J Trauma 31, 44-50.

30. Grewal R, MacDermid JC. The risk of adverse outcomes in extra-articular distal radius fractures is increased with malalignment in patients of all ages but mitigated in older patients. J Hand Surg Am. 2007 Sep;32(7):962-70.

31. Voigt C, Lill H (2006) Welche Vorteile bietet die volare Plattenosteosynthese gegenüber der KirschnerDrahtstabilisierung bei distalen Radiusextensionsfrakturen des alten Menschen? [What advantages does volar plate fixation have over $\mathrm{K}$-wire fixation for distal radius extension fractures in the elderly?]. Unfallchirurg. 109(10):845-6, 848-54. German.

32. Shyamalan G, Theokli C, Pearse Y, Tennent D (2009) Volar locking plates versus Kirschner wires for distal radial fractures--a cost analysis study. Injury. 40(12):1279-81.

33. (2021) Euro to U.S. Dollar Spot Exchange Rates for 2009 from the European Central Bank [Internet]. The Pound Sterling Live - Today's Rolling Coverage of the British Pound Sterling. Available from: https://www. poundsterlinglive.com/bank-of-england-spot/historicalspot-exchange-rates/ eur/EUR-to-USD-2009

34. (2021) Staff USIC. Inflation Calculator: Find US Dollar's Value from 1913-2021 [Internet]. US Inflation Calculator |. 2021. Available from: https://www.usinflationcalculator. com/

35. Özkan S, Westenberg RF, Helliwell LA, Mudgal CS (2018) Distal Radius Fractures: Evaluation of Closed Reduction and Percutaneous Kirschner Wire Pinning. J Hand Microsurg. 10(3):134-138.

36. Burgess L, Kynoch K, Hines S (2019) Implementing best practice into the emergency department triage process. Int J Evid Based Healthc. 17(1):27-35.

37. Combined Randomised and Observational Study of Surgery for Fractures in the Distal Radius in the Elderly (CROSSFIRE) Study Group, Lawson A, Naylor JM, Buchbinder R, Ivers R, Balogh ZJ, et al. (2021) Surgical Plating vs Closed Reduction for Fractures in the Distal Radius in Older Patients: A Randomized Clinical Trial. JAMA Surg. 13:e205672.

38. Huard S, Blanchet N, Leclerc G, Rochet S, Lepage D, et al. (2010) Les fractures du radius distal après 70 ans : ostéosynthèse par plaque palmaire ou broches ? [Fractures of the distal radius in patients over 70 years old: Volar plates or K-wires?]. Chir Main. 29(4):236-41. French. 